\title{
Participatory Prototyping to Inform the Development of a Remote UX Design System in the Automotive Domain
}

\author{
Stavros Tasoudis * and Mark Perry \\ Department of Computer Science, Wilfred Brown Building, Brunel University London, Uxbridge, \\ Middlesex UB8 3PH, UK; Mark.Perry@brunel.ac.uk \\ * Correspondence: Stavros.Tasoudis@brunel.ac.uk; Tel.: +44-0-747-409-3308
}

Received: 31 July 2018; Accepted: 18 October 2018; Published: 24 October 2018

check for updates

\begin{abstract}
This study reports on the empirical findings of participatory design workshops for the development of a supportive automotive user experience design system. Identifying and addressing this area with traditional research methods is problematic due to the different user experience (UX) design perspectives that might conflict and the related limitations of the automotive domain. To help resolve this problem, we conducted research with 12 user experience (UX) designers through individual participatory prototyping activities to gain insights into their explicit, observable, tacit and latent needs. These activities allowed us to explore their motivation to use different technologies; the system's architecture; detailed features of interactivity; and to describe user needs including efficiency, effectiveness, engagement, naturalness, ease of use, information retrieval, self-image awareness, politeness, and flexibility. Our analysis led us to design implications that translate participants' needs into UX design goals, informing practitioners on how to develop relevant systems further.
\end{abstract}

Keywords: user experience; remote UX; participatory design; co-creation; prototyping; automotive user interfaces; autonomous vehicles; automotive

\section{Introduction}

Autonomous driving is transforming the driving experience in the 21st-century vehicle. Artificial intelligence is core to this automation in enhancing safety and reducing accidents, although it may bring with it a loss of the traditional driving experience and the sense of being in control. This new paradigm results in a radical shift of the traditional driving experience. While part of the driving experience may be taken away by automation, travellers also have the convenience of using their free commuting time to explore different driving experiences and in-vehicle interactions in the car context. Given the possibilities of web 2.0 and the Internet of things (IoT), what were previously considered as secondary driving tasks or activities, such as interacting with in-vehicle information systems, infotainment, in-car productivity or social interactions and real-life experiences with other passengers in the car, are slowly emerging as primary activities. Previous research has investigated the non-driving-related activities that drivers want to perform while driving partly or entirely automated, and has identified the potential for mobile and ubiquitous multimedia applications in the car [1]. As a result, the recent focus of attention in automotive UX research includes design techniques for exploring automotive interaction in the drive towards automation [2]. Furthermore, understanding what it means to drive in an autonomous vehicle shows the potential of a shift from a "joy of driving" to a "joy of being driven" through the exploration of new forms of connectivity, entertainment, productivity, gaming as well as transportation-related services [3]. This space hands designers new opportunities 
for innovation, yet the deficiencies [4] entailed by the context of the car add layers of complexity to the research and design practitioners. Drawing from previous research, a critical disadvantage of the traditional context-aware methods (including Contextual inquiry, Ethnography and Cultural probes) is the effect of the physical presence of the researcher within the driving experience itself. Added to other secondary limitations, such as motion sickness of the researcher while taking notes inside the car, intrusiveness, loss of privacy, organizational challenges and effort [5], these approaches are not ideally suited to this context.

Previous attempts to mitigate some of the domain-specific deficiencies and support designers in the exploration of new user experiences have led to driving simulator platforms [6] that support the rapid iterative development of in-vehicle user experiences. Other research attempting to explore this design space "in-context" has led to new methods and tools, such as "trip experience sampling" (TES) [7], a context-aware low-tech method of remote user experience research in the car that addresses the immediateness and situatedness of automotive user experience research. Similar tools designed by Niforatos et al. [8] address in-situ measurement methods and avoid the disruption of users, a limitation for which TES has attracted criticism. Their work introduces EmoSnaps, a mobile application that captures pictures of facial expressions unobtrusively throughout the day and uses them for the later recall of momentary emotions. More recent research [9] has introduced systems that attempt to make sure that the in-vehicle automotive interactions can be designed, tested and understood before mass production, inspired by previous knowledge in ubiquitous computing and remote user experience research systems including "Momento" and "Dart" $[10,11]$ to support the holistic understanding of a safe driving experience. We therefore see the emergence of a research agenda attempting to overcome the difficulties inherent in the setting and which can capture the situated context holistically, by developing supportive user experience design systems to meet the needs of the automotive UX designers.

The needs of UX designers in any domain have been the subject of debate within the research community. Much of the available literature on experience design is in line with the needs that TES is addressing, by supporting the capturing of an experience, momentarily, when it happens [12]. Other researchers [13], however, disagree that UX is a momentary emotion, and the designers need to capture it, evaluate it with psycho-physiological measurements, before later designing for it. Here, it is supporting the long-term user experience that matters, rather than the momentary emotion that could even be meaningless to the user. Other recent work explores eudemonic user experiences (contrasted with hedonic experiences), in accomplishing personal goals through technology use [14]. This orients to technology design in supporting people's values, such as keeping up with fitness through technology. For instance, eating healthy food is hard and sometimes unpleasant, but it can serve the personal values and eudemonic goals of being healthy in the long term.

The needs of the UX practitioners when they remotely design an autonomous car are ill-defined and, to design for such needs is, as Horst Rittel and Melvin Webber proposed, a "Wicked Problem". An ill-defined or wicked problem occurs because the conflicting perspectives of the stakeholders cannot be accurately modeled or addressed using the reductionist approaches of science and engineering [15]. In our study, both the automotive domain limitations and the conflicting perspectives on how to approach automotive UX design have led us to deploy a pragmatic epistemological paradigm and to apply participatory prototyping methods.

Our aim was to identify these under-constrained needs of UX designers so that we can inform the development of a new remote UX design system that democratizes automotive innovation. The new remote UX design system is an interactive system that consists of methods and tools similar to TES, Momento, Dart, Emosnaps and Woz Way with an aim to support remote UX research and design in the automotive context. With an aim to democratize automotive innovation, the system serves as a platform for non-trained UX designers and drivers/passengers to collectively come up with the most innovative automotive experiences. Our research focused on the UX professionals' side of the interactive system and their interactive experience with it. To achieve this, we applied a 
participatory approach to design the supportive system by actively involving UX designers in the making process. We explored implications including the participants' motivation to use the supportive technologies, the detailed interactivity of the system, and the system's architecture, allowing us to translate participants' (UX designers) needs into UX design goals that serve as actionable insights for the development of a relevant system-solution. We conclude on a pragmatic interpretation of our findings to inform the design of such systems and offer directions for future work in automotive user experience design.

\section{Background}

Following a pragmatic epistemological standpoint, a critical review of previous work resulted in the collection of design guidelines and deficiencies. Following its multidisciplinary orientation, there are various domains that this primary knowledge is derived from and this also allows us to identify conflicting perspectives. As a result, there are no set requirements for the design of a compatible remote automotive UX system, but we can identify a set of guidelines. We highlight the most relevant guidelines from previous work in the three research areas identified: the UX design process, the design of remote communication systems, and design deficiencies identified in automotive systems. These guidelines were used as stimuli in our participatory design methods to initiate discussion and action around them. They were then also used to design the components of a toolkit used in the early stage of our participatory design. We present some of these guidelines in the following subsections.

\subsection{UX Design Process Guidelines}

User experience designers follow different methods and processes when designing for interactive experiences. Following on the most relevant manifestations of design methods and processes, we used Design Thinking approaches (IDEO) [16], at the early stages of the design cycle. As a result, the design of a supportive system regarding tasks and processes should be able to help the designer through the understanding of the challenge, the preparation of the research and the gathering of research inspiration supporting the "discovery" cycle. The approach also involves storytelling, the search for meaning and the framing of opportunities in the "interpretation" cycle.

Previous researchers have also mentioned the need for insights into the emotions of the users. The fact that consumers are "feelers" as well as "thinkers" [17] suggests a need for supporting the identification of emotions in user behavior. A system that supports the user experience design process according to Hassenzahl and Tractinsky [18] will need to support the designer to identify the user's internal state and the environment and the context when the interaction occurs.

Contextual understanding is undeniably a critical need for user experience designers. As previously mentioned [19], the social environment of the interaction, the physical environment (where the space of the interaction is dynamic or static), and the time-dependence of the interaction in a specific situation, should be observed by the designer to spark inspiration or to help them in gathering insights into and achieve a deep understanding of the user. Supporting the mapping of the context is both of great importance to a holistic understanding of the interactions taking place and necessary in designing for people and their experiences while involving them [19].

According to Whiteside and Wixon [20], productivity and learnability are not experienced as primary features of use, and thus the system should support the identification of the user's experience at the moment that they experience it. Other research highlights finding the right timing for communication or interruption [21] when exploring behavior in its context to favor intrusiveness avoidance at the expense of time-dependence. Nevertheless, there is controversy surrounding the time dependence of contextual understanding when designing for UX. Some researchers suggest supporting the previous process of understanding the user immediately after the interaction when the situation occurs [22], while others [13] suggest supporting the long-term understanding of context to help design for the overall UX. 


\subsection{Remote Communication Guidelines}

When designing for remote systems, the social context of the communication and the medium of communication are considered of great importance. Context has been found to influence a communication medium's perception and effectiveness [23]. In a remote communication, this can be frustrating as social appraisals are more likely to play an important role when others are present than when absent [24]. For instance, a person may perceive a communication technology as inappropriate because his or her friends or family who are present were intimidated. As a result of these abstract social, moral codes, systems are recommended to support the avoidance of disruption in situations of co-presence [25].

As previously highlighted, choosing the medium for a remote system is controversial and dependent on the context. Different media are better equipped to disseminate information ("conveyance") while others are better at engendering mutual understanding ("convergence") [26]. Guidelines on previous research suggest that media used in remote systems should support the avoidance of Face-threatening acts (FTA), including acts of criticizing, disagreeing, interrupting, imposing, asking favors, making requests, and embarrassment [27]. In addition, systems should support etiquette in communication to make social interactions more pleasant and polite [28] by using the appropriate medium.

A system's effectiveness also depends on the medium used. To support UX designers in observation of a hidden or latent need, the communication medium used should support the identification of cues such as frustration, confusion, or unexpected usage. Other guidelines on the communication media and the effectiveness of a remote system include the importance of social "presence". These studies suggest that, to communicate effectively, one should match the social presence of the medium with the level of personal involvement and attention of the communication task [23]. For example, when the medium allows teleconferencing, employees are more aware of others' status and reactions, and they will be more cautious about their self-image and behaviors [29].

\subsection{Automotive Domain Deficiencies}

When designing for interactions, designers need to attend to the context, i.e., the social, physical and temporal environments of the interaction, in a given situation. Any of these aspects can have a direct effect on the interactive experience. Designers and researchers $[5,19,30]$ generally agree that context is both of great importance to a holistic understanding of the interactions taking place and necessary in designing for people and their experiences. However, in automotive studies of Human-Computer Interaction (HCI), there are few studies (with some exceptions, e.g., [31-33]) that have previously investigated the influence of the automotive context in a systematic way using qualitative methods in real time driving situations, based on a holistic research approach. Ethnographic research in the automotive environment is unconventional; nonetheless, recent studies have used it to explore sociocultural issues in the driving experience in collaboration with Nissan [34]. Other Companies including BMW have even undertaken early remote open innovation and co-creation paradigms [35] involving people in the generation and the design phase. Meschtscherjakov et al. [5] highlighted important limitations for researchers and designers in the automotive domain. At a higher level, supporting the holistic understanding of the automotive context dictates that we mitigate these limitations. Previous research [4] has summarized the deficiencies which affect automotive design and communication. These include the distortion of the experience by the physical presence of the researcher in the car, intrusiveness, minimization of the effort of traditional contextual methods, the avoidance of cognitive effort, as well as concerns for the safety and privacy of the driver and/or passenger(s) while the interaction takes place.

Finally, we cannot neglect the fact that the communication involves two people, a researcher/designer, and a driver/passenger. Designing to support this communication also relies on the perceived trust, control, ease of use, enjoyment, attitude towards the methods and tools used to 
interact and the intention to interact, of both the researcher/designer and the driver/passenger and we therefore add them to our set of limitations.

\subsection{Remote UX Design Systems and Automotive}

In user research, the two main activities are observing and interviewing participants as seen in (see Figure 1). User experience researchers are interested in accurate information about people, their tasks, and their needs, and, since interviews are not always accurate, observation has greater value. Observation of human behavior is an essential element of most user-research, including usability testing, contextual inquiry, naturalistic observation, shadowing, covert observation, and participant observation. The main differences between these methods are the location of the observation, the amount of interaction with participants, the proximity to the participant, and the participants' knowledge of being observed [36]. To achieve unobtrusive results, practitioners use a variety of tools [37] in UX Research and Design UX researchers need to share their inspiration material, data or insights using synchronous or asynchronous communication tools. Sketches and discussion of the ideas can be supported using a combination of tools such as Google Drive or InVision and Skype or Zoom. The simplest solution is to ask team members to sketch on their own, then take photos of their sketches and share them on Google Drive or InVision, so everyone can see them while discussing the problem via Skype or Zoom. To understand the problem of interviewing or communicating orally, a variety of Web conferencing tools, including WebEx, GoToMeeting, and Zoom, can support UX designers. Finally, screen sharing is more effective than any shared drawing tool. Just as UX designers come from a variety of backgrounds, they like a variety of different tools that they use when designing user experiences.
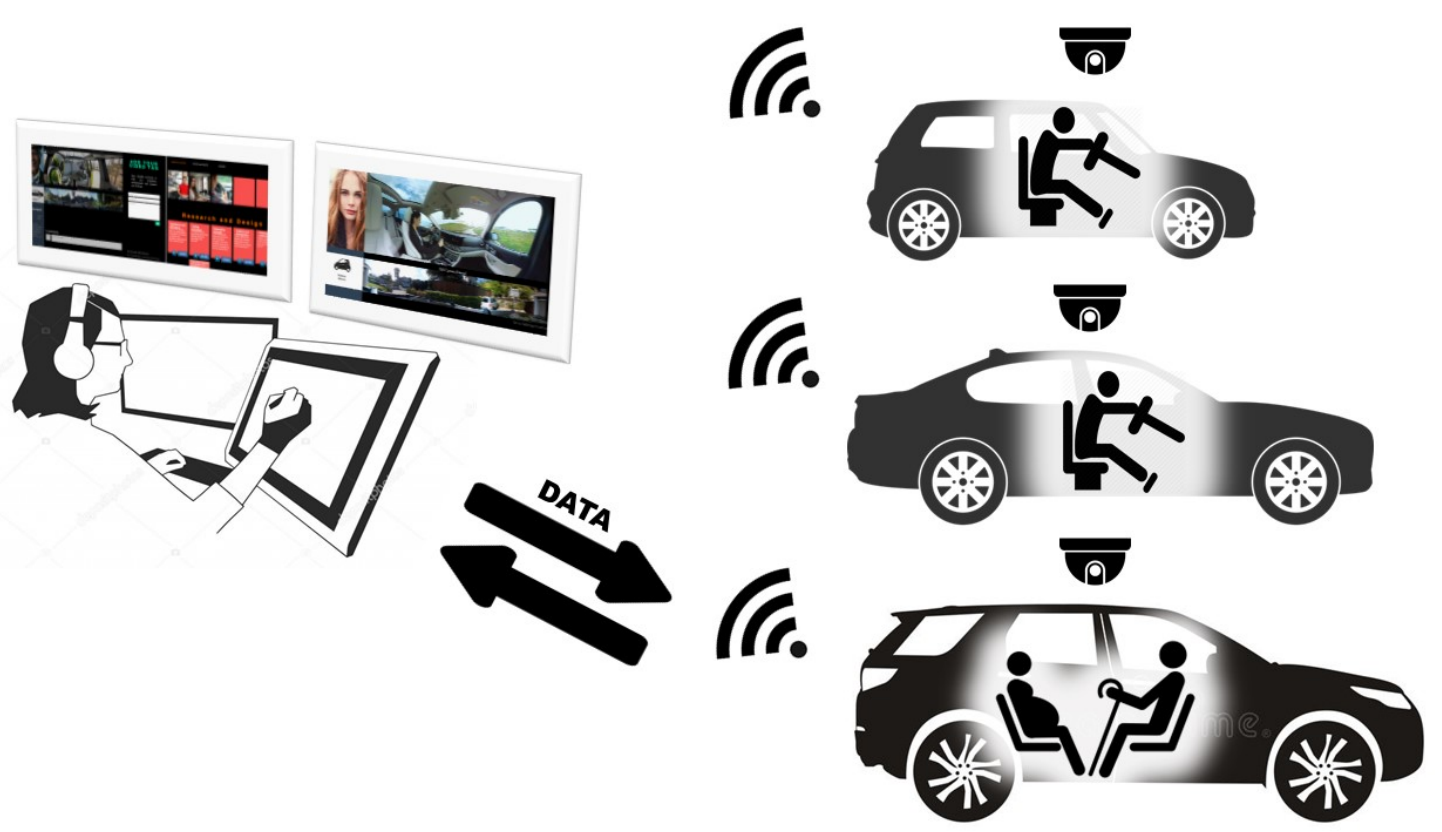

Figure 1. Remote automotive UX systems support in UX research and design by observing; interacting with synchronous or asynchronous data concerning the context of the car; and communicating in real-time with the passengers or the drivers.

Automotive researchers use remote observation techniques initially applied in the mobile phone domain since similar systems in the automotive domain are not fully developed to the extent that supports both UX research and design. Previous researchers [38] introduced toolkits that use Android smartphones to offer a low-cost, manufacturer-independent and scalable in-car agile prototyping and research environment. CarDaT (Car Data Toolkit) enables researchers to gather data on human 
behavior and designers to create novel context-aware interface solutions remotely by combining available remote data, smartphone sensor data and other car generated data. Even though these toolkits are not part of a complete system proposition which also supports interviewing, as a fundamental part of user research, they can still support or even replace observation and the identification of behavioral patterns of drivers or passengers. Only a few attempts have been made to extend previous remote systems, foreshadowed by Carter et al. [11], by providing real-time video and automotive data with the ability of designers to directly interact with a driver through speech and in-car interfaces. WoZ Way [9], for example, allows designers to watch the real-time driving experience via high-fidelity video and audio, and also simultaneously receive meta-data about the drive. The designer can also ask questions by using a text-to-speech messaging system, or remotely triggering custom in-car screen and electromechanical interfaces.

\section{Methods}

Even though user-centered design (UCD) has influenced our methods, our study deploys participatory design and co-design techniques to actively involve non-expert users (automotive domain experts) to the generation and conceptualization of our prototype system. UCD is a design method whose application conducts designers to develop usable design solutions for end users. Co-design, on the other hand, is a set of creative techniques whose aim is to inspire design. Creative exercises are usually applied to enhance idea generation and concept design; they are characterized by the presence of non-designers (experts) as participants, and usually led by designers [39].

As participation in this study, we refer to the term that in $\mathrm{HCI}$, as part of participatory design, means democratization and end-user involvement in the design process and it is used in the design innovative technological interventions. Previously, the use of the term within HCI research often described the involvement of people in a design process [40-43], or the gathering of insights and requirements to inform future design [44,45]. As Vines et al. described, the term participatory design generates reflection on participation in design in broader terms than if we were to reduce ourselves to tight definitions or specific traditions. They also explained how within HCI, many previous researchers describing participatory processes provide examples of working with groups who might be excluded $[40,43,46,47]$, including people with special needs in terms of health and emotional wellbeing $[41,48]$ or in contexts where the introduction of information and communication technologies might conflict with cultural traditions [49]. The automotive context—given the long tradition in different research methods and tools, which is mostly a result of the many limitations of the domain as explained in this study-does not follow the current trends in opening the design process to non-trained individuals and the gathering of insights and requirements to inform future design.

In the past, participatory design approaches including narratives, games, and artifact construction have been applied under different contexts [50]. In co-design, the participants are active design partners. The co-design process deploys tools and generative techniques [51] to access people's feelings, aspirations, and imaginations and delve deeper into the explicit, observable, tacit and latent needs of the participants. By co-designing an artifact in our study, the prototype of the system designers can gather data on what the participants say, do and make to get a deeper understanding of their needs: "One should keep in mind that the relationship between designer and user (consumer, recipient) is bi-directional. It is not as if users have well-defined requirements, which only wait to be discovered. Indeed, requirements are co-constructed in the ongoing dialog between the user and designer." [12]. In this study, our users are User Experience researchers and designers working in various non-automotive domains. They are familiar with the UX design process, but not professionally familiar with automotive contexts, as we explain below. 
As we have shown, previous work developing remote automotive UX design systems does not provide us with well-defined requirements. Consequently, we followed a top-down approach which initially capitalizes on the available knowledge of the UX design processes, automotive limitations and remote communication guidelines from previous research. Driven by our pragmatic epistemological standpoint and the nature of the problem, we apply individual participatory design workshops that actively involve participants to prototype the proposed remote UX design system and triangulate our findings. The analysis of qualitative data is our primary source to inform theory and design. Tonetto et al. [52] highlighted the fact that quantitative data are more precise and are useful for attracting investments or convincing stakeholders about the effectiveness of design decisions. This could explain, to some extent, why quantitative data are widely used in automotive research and design. Some of our participants also preferred a combination of the two.

Our methodology included the following:

- a review of guidelines;

- a design tailored to the design problem toolkit;

- participatory prototyping sessions;

- $\quad$ analysis of the rich data on user needs; and

- the translation of user needs to UX goals.

The use of these methods allowed us to identify users' needs and provide actionable insights in the form of UX design goals to help practitioners in the development of relevant systems.

\subsection{Toolkit}

Participatory design tools and methods are widely used to share control, share expertise and get inspiration for change [49]. Participation through co-design has attracted the attention of researchers in HCI who need to gain rich insights into the explicit, observable, tacit and latent needs of the participants [30]. In line with this, McCarthy et al. [53] suggested identifying the uniqueness of the individual's experience by eliciting the salient situational circumstances.

Previous research [54] summarizes the terminology used to highlight techniques and toolkits when one uses a participatory design method. It describes a method as a collection of the material components (toolkits) and techniques that are used in combination with participatory design activities to serve a specific purpose. Previously, Sanders et al. and Sleeswijk et al. used participatory design toolkits [54,55] and Pettersson et al. [2] used participatory design techniques in co-design and co-creation activities, to achieve higher active involvement of the participants. In some cases, previous researchers even delve into the latent explicit and observable needs of the participants when using them. Sanders [56] explained that, to gain insights into experiences, thoughts, feelings, and dreams, we should provide the participants with tools which are focused primarily on what people make in addition to what they say and do. To serve this purpose, we designed and applied a participatory prototyping toolkit as part of our method.

We based our prototyping toolkit on the Design Thinking (DT) model for designing new artifacts. The toolkit consisted of low-fidelity representations of: (A) the processes, which involved three basic screen-wireframes representing the three steps of the process used to support the need for discovery and interpretation; and (B) the available components, including communication technologies and interactive elements. Two previous low-fidelity designs served as stimuli material for the co-design workshop. 
The first part of the toolkit consists of cards of low-fidelity paper illustrations of components (see Figure 2) derived from the guidelines of previous work. These cards both serve as stimuli for discussion (i.e., what the users say and do) about the design and application of the system in context, the acceptance of the technologies used, the system's architecture and interactivity, and lay the foundations as a tool for prototyping (what the users make). Similar tools presented by Sanders' "make" tools [51] are commonly used by practitioners, including the "interface toolkit" by Frog design. Components include the following: car passengers ( 1 or many); behavioral tendencies; relation (friends, family, and intimate other); emotion, skills; physical and temporal context; where (map); when (time and date); weather (sunny, rainy, etc.);temperature; noise (very loud, not loud at all, etc.) system context; percentage of use of in-vehicle information systems (IVIS); video/audio/text/emoticons/gifs; charts/pies; snapshots infographic; storyboard; and customer journey.

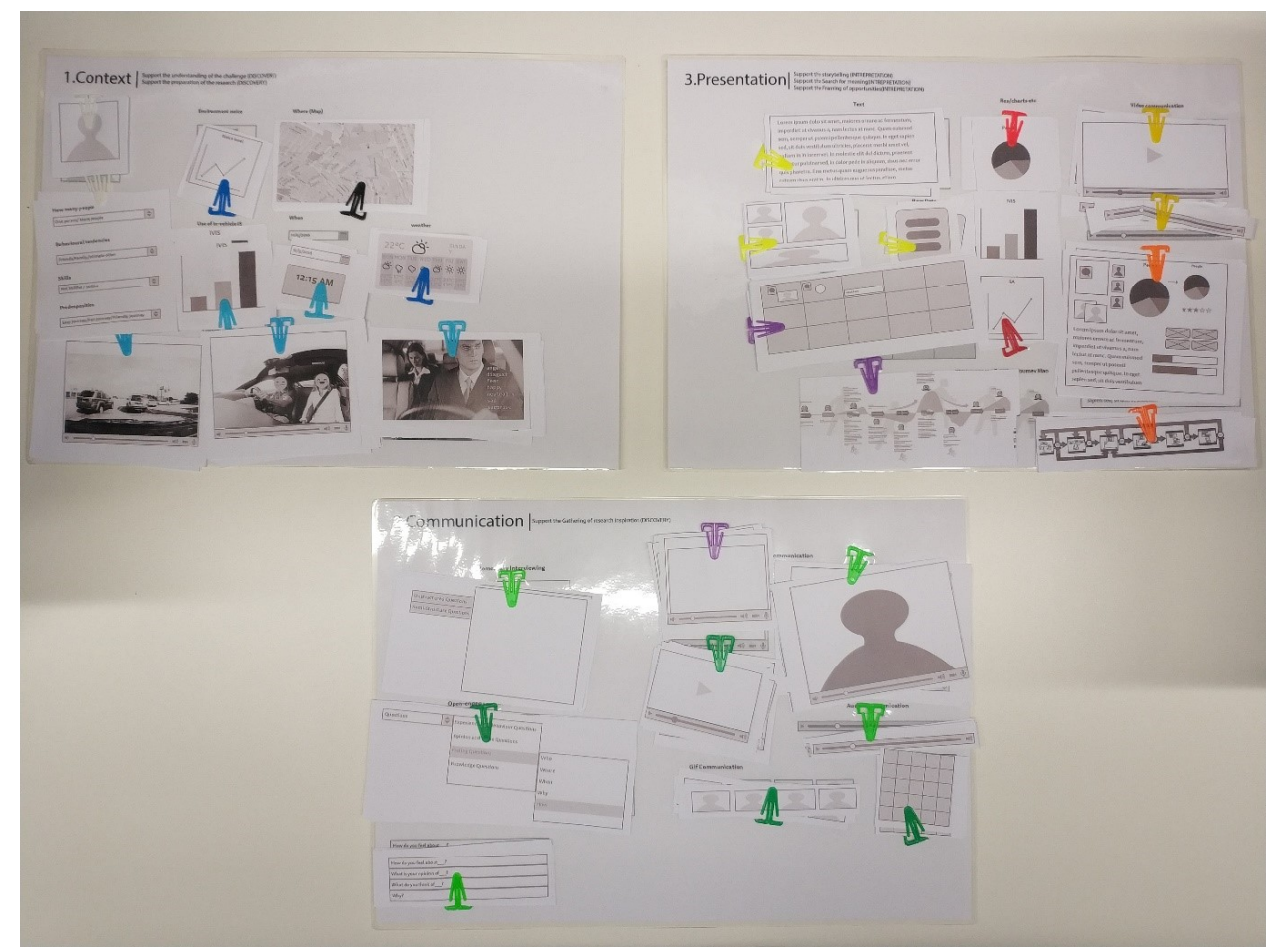

Figure 2. The paper cards represent technologies or affordances previously used to help in identifying the context of the vehicle, supporting the remote communication, and presentation. We base the selection and design of our cards on previous work on remote communication, automotive domain deficiencies and UX Design process guidelines.

Based on the IDEO's design thinking methodology, the second part of the toolkit serves the early design stages of the new experiences process. Three sheets of A3-size paper served as the space for low-fidelity prototyping of the basic-screens and interactivity for the proposed system. We designed the basic-screens as an empty web page with only a title and information on the design thinking processes. The technique that we recommended for the prototyping was to fill the basic-screens with information, notes, and the cards that we provided (see Figure 3). 


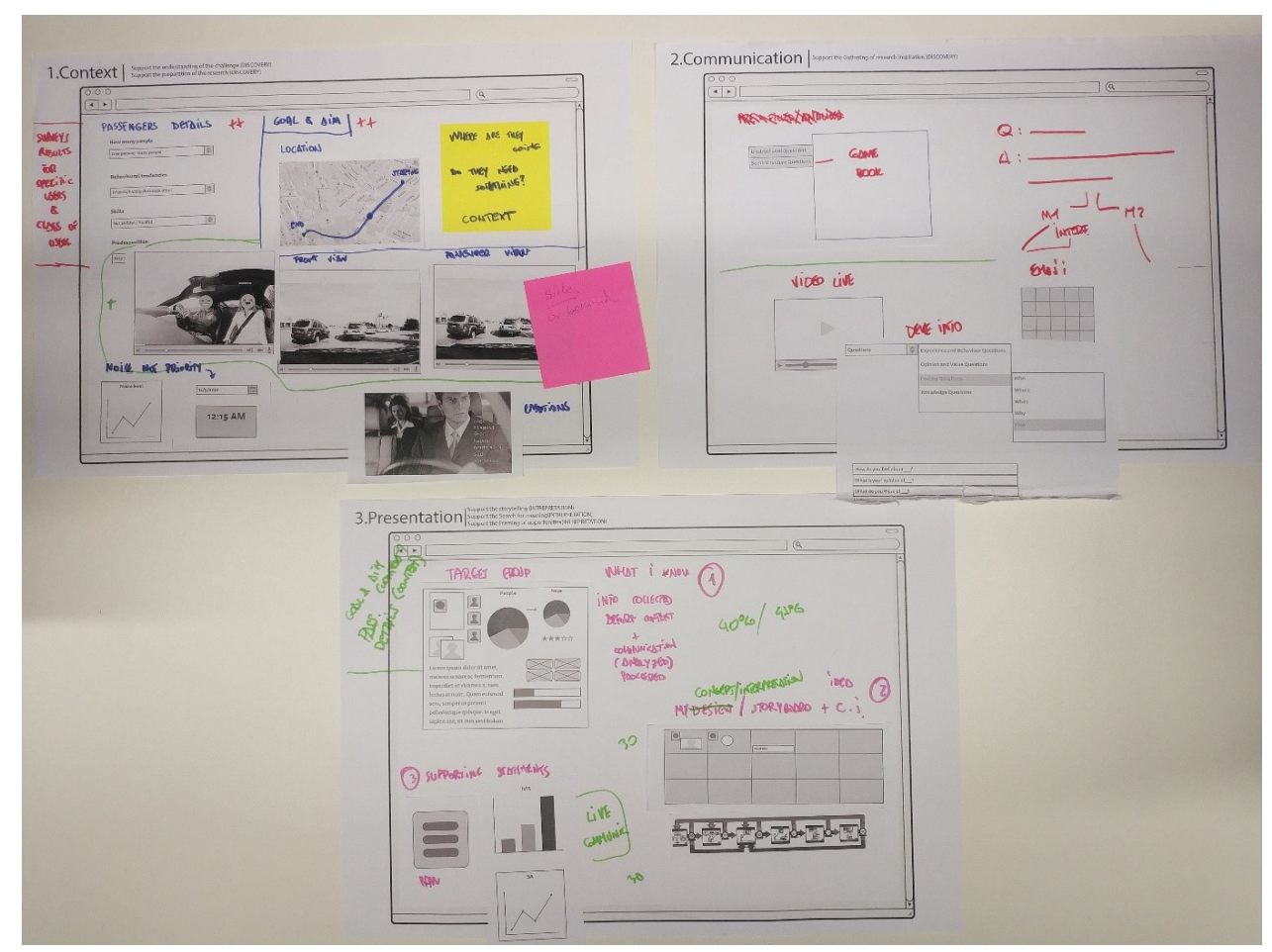

Figure 3. P6 participant's basic-screen paper prototypes of the interaction. The three basic screens represent the early stages of the design thinking process. UX researchers/designers individually co-designed their versions based on their needs.

\subsection{Participants}

Iversen et al. [57] argued for a value-led participatory design approach. They saw a co-design process, at its core, as a negotiation of values that all participants bring to the table or which emerge from a collaborative experience. It is not only which values are important, but also whose (participants) values drive the design process. To assess these values, we chose to involve UX designers who were not directly involved in the automotive domain. The democratization of UX design in automotive has two ends. The first one is to actively involve drivers and passengers in the design process. The second and most important, since it is the focus of this study, is to involve UX designers (not trained in the automotive context) in the automotive co-design process. It is in the interest of this study not to fixate on pre-conceived views of what are the right methods and tools based on Automotive UX designers since our end users are not necessarily UX designers with automotive design experience. Furthermore, our conversations with designers inside the automotive industry suggest that the industry is extremely traditional regarding the methods and tools used to design and evaluate interactions in comparison to other domains. To achieve a state-of-the-art result and an innovative outcome, we wanted to avoid traditional thinking, or recreate existing or obsolete solutions. Since the system itself aims to provide support not only to expert users such as automotive UX designers but also to general UX designers, this decision fits our purpose.

We recruited 12 UX professionals aged 18-34 years. These consisted of eight UX designers/researchers working in academia and four in industry, recruited from a "snowball" referral of seven initial participants. All participants had expertise in design and high familiarity with new technologies. The workshops were individually assessed. These were all holders of a driving license and were therefore non-professionally aware of the driving experience and driving context. The intention of this was to secure the holistic nature of our design, and to make sure that we did not exclude other stakeholders' values such as the drivers' values from the design result. Each study participant received a reward/token and a box of chocolates for their time and effort. 


\subsection{Participatory Prototyping Procedure}

To identify the values of the designers that need to be fulfilled, we used prototyping workshops to collect rich data on what the participants say, do and make and to translate them into design goals. "Prototyping is a venerable system development methodology that involves construction and test of prototypes of systems, often for purposes of clarifying vague requirements and often in collaboration with the prospective users." [58]. Participatory design limitations including recruitment limitations, and time and location sensitivity dictated that we actively involved participants in individual sessions. Furthermore, individual sessions were useful because the system being designing was relatively new, and therefore under-constrained. Using group sessions would have undercut part of these insights. In contrast, in comparison to the group sessions, the results were more detailed and personalized due to the time spent with one individual at a time.

In each session, the participants co-designed a low-fidelity prototype of the front-end interactions of the system using the components that were previously provided to them. To conduct the paper prototyping along with the cards of the components, we provided sticky notes, a marker, pencils and glue for the participants to construct their three basic screen-wireframes. We encouraged them to use whatever means they felt were most natural to them and in many cases, we constructed meaning with them collaboratively.

The sessions were conducted similarly to a group session with one researcher as a moderator who is also involved in the discussion and the co-creation of meaning and content without leading or biasing participants. When the moderator is one of the artifact designers, they should be cautious not to introduce any personal bias into the presentation of the artifact. We tried to avoid bias on a prototype design since we provided the participants with the basic platform and raw materials that were then used to obtain their prototypes.

An introductory video of a physically present designer interviewing a driver while they are driving in a real-time driving situation was displayed before the workshop started. This was to inform UX designers of the current field methods used in Automotive Design and Research and to allow them to empathize with the automotive deficiencies mentioned above. Sessions lasted 50-60 min, with approximately $15 \mathrm{~min}$ for each of the three tasks to which the participants were assigned. We were mainly concerned with capturing "how" and "why" the participants would like to be supported in the discovery and interpretation phase in the early stages of the remote automotive UX design. Observation and semi-structured interviewing took place beside other complementary methods, such as co-designing the artifact. Observation, without following a specific observation scheme, was mainly a tool to capture the "do" and "make" data other than what they say. Notes of critical points were taken to support the findings and video/audio recordings of the sessions were taken for later analysis. At the end of the participants' tasks, we used a semi-structured interview to ask participants to identify the following:

- The contextual data that would support the UX designers in designing for people's "driving experience" in an autonomous vehicle. When we say "driving experience" in an autonomous vehicle, we mean understanding and designing mostly for secondary activities and interactive experiences in an autonomous vehicle including infotainment, productivity, and gaming or other digital services.

- The tools and techniques they would use for in-depth communication and information elicitation; what existing tools and techniques might support synchronous and asynchronous communication.

- The tools and techniques that would support them in communicating their results to other stakeholders; how to support them in presenting their rich findings. 
Based on good interviewing practice, we used complementary "why" questions to shed light on short or unarticulated answers. We encouraged the participants to provide critical comments about their choices and designs. Furthermore, we prompted discussion by asking them to structure the information, the main groups of the content and add any other components that are not present in the stimuli material. As a result, we have captured rich data of what the participants say, do, and make, using a combination of observation, momentary qualitative interviewing and video records of the interaction. We also gathered the artifacts of each session and analyzed them to support the findings.

\subsection{Analysis}

After the participatory design sessions, we implemented an inductive approach to data coding and analysis. We transcribed and coded the audio-visual data. Thematic maps helped us to sort the transcripts into related groups from which we later drew our themes. However, it is impossible to be purely inductive and completely ignore the semantic content of the data when we code for a particular theoretical construct. What is essential in this first part is that we prioritized the participants' meanings. We concluded on the user experience designer's values that need to be fulfilled when we design such systems. These findings are presented as the needs of the UX designers in the automotive context and support the summative theoretical contribution which aims to evaluate such systems.

The prototypes created are the means that the participants use to express their multi-layered needs and the experiences they want to have when interacting with similar systems. Therefore, we can extract more in-depth information from the explanation of the created prototypes and even relate them to the previous data. Consequently, we go beyond the participants' meanings and the themes that are derived from our analysis and suggest a pragmatic interpretation of the UX Goals of the UX Designers based on context mapping techniques which include the illustrations of the artifacts. Affinity diagrams [59] and context mapping techniques [30] are both techniques that analyze rich qualitative data. They both use coding of participants' quotes or notes, and they both try to identify patterns or clusters of behavior or activity. They both use big spaces as tables or walls to map, structure, and cluster the previously collected data and extract meaning. We achieved a holistic understanding using these techniques (see Figure 4) to triangulate the video and audio transcripts (what participants say and do) with the paper prototypes (what participants make) in the second part of the analysis.

Approaches for visual element analysis in isolation, including Zmet and Kansei, were previously explored [30] without satisfactory results. More information is found in the stories of the participants and their relationship with the visual elements, in our case the prototypes. The participants' hidden or latent values that needed to be fulfilled based on our findings were later translated into seven UX goals. UX goals, as previous researchers explained [60], support designers in developing products or systems. In this study, these findings support the formative practical contribution to the design and development of remote UX design systems in the automotive domain. Nigel Bevan [61] also highlights how important it is to establish criteria for UX/usability goals at an early stage of design and to use summative measures to evaluate whether designers achieve them during development. 


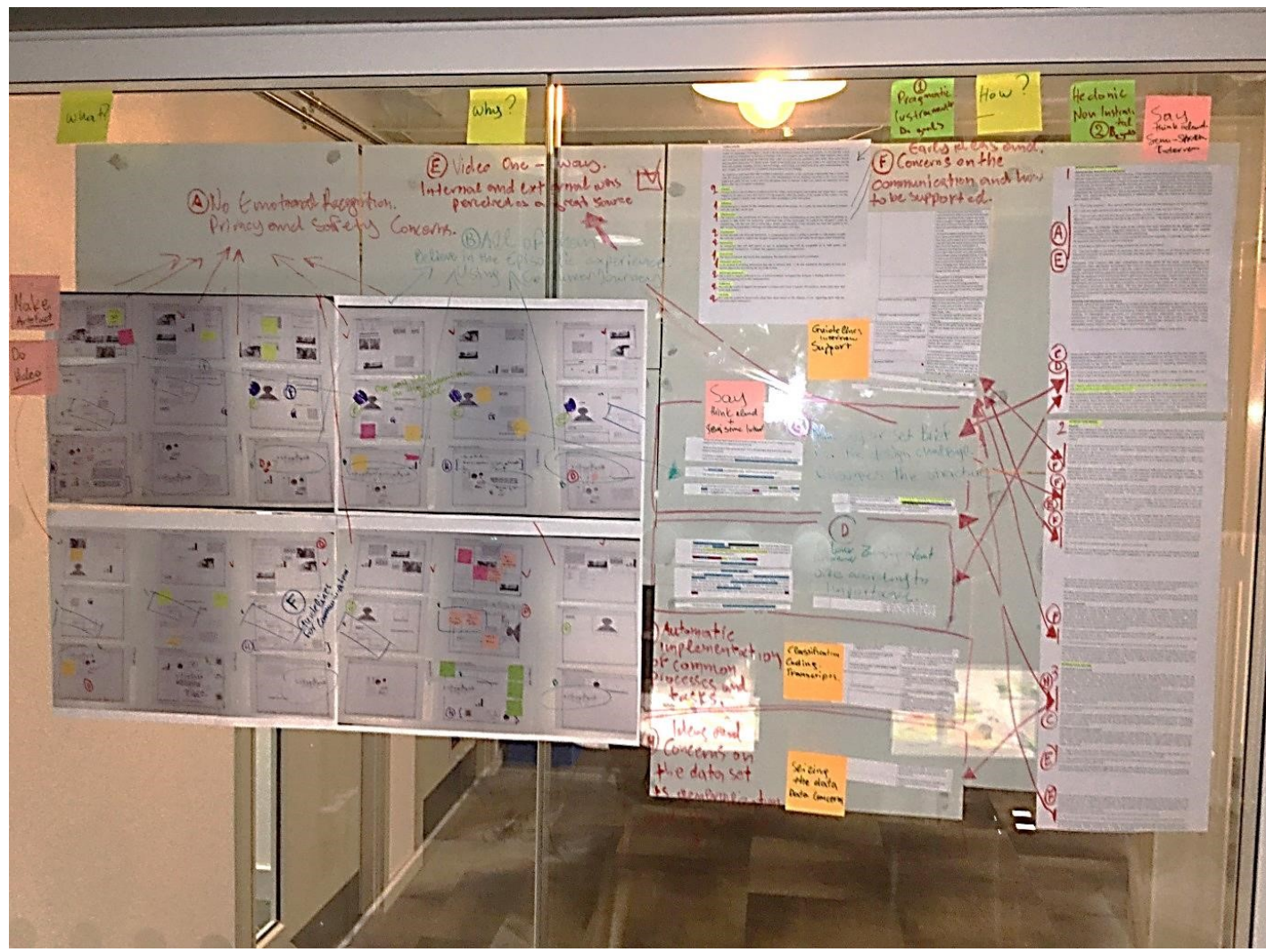

Figure 4. A glass wall was used to map all the information available, identify patterns, and cluster the critical issues concerning the prototypes. Ten needs and seven UX goals emerged from this analysis.

\section{Results}

The process of developing and validating these artifacts identified numerous areas where future research is required. Using the knowledge gained from this study will provide insight into what researchers might look for and what they might see when studying remote UX design systems in the automotive domain. We collected digital audio and video data of the workshop and analyzed the transcripts and the field notes using color coding and thematic maps. We mainly coded repetitiveness of a phenomenon or a participant's opinion, commonalities, and relationships with other participants' views and actions, and patterns of behavior. Grouping and naming the themes, we prioritized the participants' meanings and concluded on the participants' values that need to be fulfilled when we design such systems. Although most of the themes express non-instrumental hedonic or eudemonic values, some themes are purely instrumental and pragmatic including the "contextual components" and "preferred communication medium". As a result, the findings are structured and presented in themes. We examined the role of a set of values that we identified in this study and aimed to develop a remote participatory UX design system. The first theme examines the role of intrusiveness, emotions, and behavior, followed by usability and information architecture and their impact on the effectiveness of our system. The third theme of the findings presents the value of the quality of communication and the fourth designer's empathy about their end users. The last themes examine the role of context, and the preference of the participants with regard to the communication medium of our system. Below, we present a summary of the needs and how they relate to the UX goals that are derived from our analysis.

\subsection{Intrusiveness, Emotion and Behavior}

The first theme that emerged was Intrusiveness. Even though some participants were more enthusiastic about the various supportive technologies that we provided as stimuli, the majority 
expressed a general mistrust of the effectiveness of using emotion recognition technologies to support design. The fact that emotions could not rely only on facial recognition and the fact that emotions can be affected by different factors when users are in social settings leads designers to a develop a mistrust of the technology. Additionally, even though the participants understand the possibilities of the technologies as mentioned above, ethical considerations such as privacy and safety were expressed as they see themselves as potential users. Thus, we mainly avoided face tracking and similar technologies.

P1: "For safety purposes" "You can see children inside the car, and the passengers are not properly protected."

P1: "I am not really attracted by this kind of information. For the same concern. Privacy."

P5: "I am not sure what one sees in your face is what you feel, I think there is a discrepancy there. It is really difficult to understand emotions just from the facial expressions", "my facial expression can be affected by so many factors." On the contrary, the behavior of the users in the vehicle was more important for the designers than emotion recognition. The participants suggested alternative, less intrusive methods such as observation, empathy building, and technologies such as video or audio monitoring.

P6: "I would probably like to see him. If I was doing the interview remotely, I would like to see how he reacts to the questions that I ask and to different situations."

P6: "If you have a 360 view of everything around you can see the behavior."

P7: "You can design a car based on emotions, but you can also design a car based on demographics and behavioral (aspects). If someone is eco-friendly and likes nature, I could design for them but I cannot do the same with emotions."

The participants provide evidence that other technologies are more useful in designing for experiences. More specifically, technologies such as video or 360-degree view of the environment are suggested. A possible explanation for these results is that the context of the person interacting in the car is more important than the individual and can generate insights or inspirations for the design of the new automotive experiences. Even though the designers avoid monitoring facial expressions through technology, paradoxically they would like to see the users interact with their environment and want to observe their reactions. That is possible because of the identification of cues such as frustration, confusion, or unexpected usage, which show a hidden or latent need as we mentioned previously in this paper. The fact that designers empathize at all times as drivers themselves can explain this reaction. Therefore, ethical considerations of private information lead them to decisions of mistrust of specific communication mediums in comparison to the rationale of the media richness hypothesis [62].

\subsection{Usability and Information Architecture}

Regarding the information architecture of the system, participants had various useful suggestions and ideas, and many usability qualities were highlighted as necessary by the participants. Although some of the participants wanted to include all the possible information they found on the system's toolkit, most highlighted the need for a minimalistic design approach. Thus, participants avoided redundant or complementary components regarding information content and architecture. Even though the hierarchy of information was generally not considered essential, participants used patterns of content-architecture to group content and brought essential components in the front of the interaction, avoiding an utterly loose interaction with no hierarchical structure. Even though sometimes the complementary information occupied most of the free space of the basic-screens, participants mainly followed the rule of bigger equals more critical, enlarging the more critical information.

P1: "You want punctual information really direct, visual maybe audio I think is really effective."

Moreover, they highlighted the need for a flexible design that adapts to their needs each time they interact with it. Immediateness is another quality that the participants stated as crucial for this type of interaction. The participants want the information to be immediately available to them. Finally, Information retrieval and ease of use were reportedly found to be crucial for our system, with the participants being concerned both by the "hustle" of the interaction and the possibility of forgetting information and missing essential findings throughout the process. 
P6: "I don't think there should be any hierarchy in the position of the visual systems. If I am blue sky and I don't know what I am doing I would choose this hierarchy though."

P1: "With emoticons, you have a question and a list of emoticons that the driver can reply immediately."

P5: "Maybe what is very useful you can star it and it can get down to the 20 per cent. If you think it is important and you like it and not want to forget about it, why not?"

The designers preferred flexibility in the way they are going to use their tools as part of the system. The need for exploration and experimentation is fundamental in the early stages of the design process, and that could be one explanation about their preferences. Another explanation is that they do not have a predetermined set of scenarios, but they depend on the observation to identify the behavioral patterns of the users. It seems possible that the designers need some initial time to grasp the interaction scenario, identify the preferred contextual information and then decide on how they want the system to support them accordingly.

\subsection{Quality}

Participants expressed concerns about the quality of the communication being affected by trust. When the system supports them in communication with the driver/passenger, it is vital that the used technologies support a trustworthy communication interaction for both parties. They generally believe that when there is a lack of trust, the goal of the designer is undermined. Accordingly, they propose a more natural, transparent and socially present communication to mitigate the feelings of mistrust to them and the system by the passengers/drivers.

P7: "If there is no point between you and the user, It is going to go downhill from there because you will never understand each other, that should be established. Building trust is the first thing. I want unstructured (interviewing) for the same reason."

P8: "Having an audio of me or of another person for self-presentation issues doesn't make much of a difference. If it wouldn't be my voice and it was somebody else's I don't want it to sound artificial. That is a very bad idea. I prefer a terrible natural voice than an artificial one."

Participants are also concerned about the quality of communication affected by self-presentation issues. Their appearance can affect the level of engagement with a communication interaction that is going to take place. The same effect also works vice versa, i.e., when people are distracted by the image that the designer communicates towards them and change their responses and behavior accordingly.

P5: "I don't want to be good looking in case they are looking at my face, and start thinking about other stuff rather than design. We don't need to have the same interaction every day. We can have different types. We can see each other today. If I am not presentable. If I have an initial video it can work and then you can have another type of communication."

P12: "They cannot see the designer. Because the answer can change. If I see your facial expression, then my next answer is going to be different. For example, you are asking me how is research going and I am responding I didn't do my literature review and you are laughing. Your next question if you ask me how often do you see your supervisor, I might say every week. (very often)."

Finally, the participants feel that a structured way of communication feels like a duty and a preferred semi-structured manner will give them more feedback. On the bases of instantaneous communication and face-threatening acts, they are also concerned that they may lack the skills to provide high-quality communication and that they need to be supported by the system to achieve the levels of quality that are expected by this kind of qualitative conversations with people.

P9: "People react in a way you react towards them. If you yell at someone they will probably yell back at you. The driver is going to see you sometimes. If he is going to make signs you can see him. Human beings respond to the way they are being treated. I would strongly lean on that the designer should be trained and experienced."

P11: "Unstructured (communication) needs an experienced researcher."

The results here are in agreement with those obtained by previous studies about the self-image of people using remote communication technologies. A possible explanation is that the participants are trying to protect themselves from an unexpected situation and at the same time to protect the validly 
of the insights gained by the interaction with the driver/passenger through the system. The fact that they do not want their presentation to affect the interaction shows again empathy for the people with whom they are going to be interacting.

Building trust and maintaining engagement is again expressed as a desire here by participants. The medium that can serve these needs of the designers is presumably the most useful as well. It is worth highlighting here that the effectiveness of the medium in use is not a primary concern of the designers.

\subsection{Empathetic Design}

On many occasions, the participants empathized with the user and explained their decisions based on the experience that they would like the driver/passenger to have in this two-way interaction. Their values and their company's values (when applied) lead them to a definition of the appropriate interaction. They are concerned with the perceived safety, comfort, naturalness, politeness and the feeling of being valued or any possible frustration that the drivers or passengers will experience during this interaction. The participants consider it essential that the driver finds himself in control and that the communication medium that they are going to use is appropriate for the situation.

P4: "Create an environment where they feel safe because it is a radical change."

P9: "Structured feels like a duty. If someone is relaxed, he or she tells you more. A bridge between being polite and having all the information you need."

P12: "Unstructured questions allow a better user experience because people like their opinion to be heard."

Participants highlighted the need for the naturalness of the communication, possibly prioritizing the sense of more informal and natural communication. Empathy is expressed here by many participants. One of the participant's claim provides a possible explanation: the insights are going to be more in-depth if the person feels more relaxed. Another explanation is that they are already "walking in the driver's shoes" and feel that they would not like a cold closed and structured interaction.

\subsection{Contextual Components}

Participants generally expressed the need for contextual data that they can relate to, at the time, when the driving experience takes place. The place and the environment also shape the experiences of people and are equally important. Participants find the social context of the drivers to be of great importance to a deeper understanding of the situation. Finally, long-term patterns of behavior are thought to be of help to designers in identifying opportunities before they delve into a more in-depth understanding of the situation.

P5: "You need to know if its rush hour in the morning and you have to get your kids to school or if it is a bit later in the day."

P8: "It goes back to the personality depending on different things. With the personality, If you have your girlfriend in your car or someone you really care about, you might be driving a little bit more carefully, but then if you are with your first date you might want to impress her and drive more dangerously. And you might want to identify a pattern based on that."

P6: "Otherwise how can we improve the commodities of this family here maybe you need data that are collected over a few months."

The majority of participants agreed on longitudinal behavioral data and supporting rich contextual information as a source of inspiration for the design of new automotive experiences in autonomous cars. A possible explanation is that they want to design for long-lasting, meaningful experiences in comparison to momentary hedonic experiences [14].

\subsection{Preferred Communication Medium}

The primary communication medium was also a concern for the participants. A video is generally considered a vital medium towards the understanding of emotions and behavior. In such a manner, the participants' video technologies are suggested to capture behavioral patterns and to achieve more 
in-depth communication with the user. Video of the internal and external environment can give a deeper understanding of how people experience driving.

P7: "That is why I want the video. The reaction of how he is sitting, the reactions. The pattern that leads to a personality, because if someone is constantly doing something, it leads to a personality."

P11: "The external is important because we can see how he is reacting based on the environment. Sometimes he is feeling bored and sees the other way."

Furthermore, some of the participants suggested a two-way video interaction to communicate transparency and build trust among them. However, one participant expressed concerns about the importance of the video on the driver's side, basing his argument on the attention the video requires from the drivers. Many of the above qualities such as recognizing the feeling and the level of personal involvement in the communication are also expressed for only audio communication. Finally, they found text, gifs, and emoticons to be exciting means of communication but as an additional medium and not the primary medium due to the limitations in comparison to the video as mentioned above or only audio.

P2: "I would use the voice because with the voice you can perceive the feelings as well." "So if you use emoticons or text other than your voice could be less personal, so harder to build trust."

Building trust and maintaining engagement is again expressed as a desire here by participants. Additionally, participants added characteristics of utility to their decisions choosing the most appropriate medium for convergence (better at engendering mutual understanding) [26].

\section{Discussion}

Our participants' goals express their aim to fulfill specific needs. Our summative interpretation of these needs, based on empirical evidence drawn from our co-design workshops, informs relevant theory in automotive design for experiences. Furthermore, we suggest a formative interpretation of our findings in the form of UX Goals as actionable insights. Each of these UX goals relates to one or more user needs, as presented below.

\subsection{UX Needs}

Previous work by Eckoldt et al. [63] supports the notion that meaning and positivity are related to the fulfillment of universal psychological needs (an experience becomes positive and meaningful if it fulfills a psychological need) and explores the potential of an experience-oriented approach to design for interactivity in and through cars. Identifying these needs for design and evaluation purposes attracted the focus of automotive research measuring both momentary and long-term user experiences as explained by Körber and Bengler [64], and Kujala et al. [65], consecutively. In this study, the analysis of our rich data led us to identify the following needs of our participants.

Privacy: Privacy refers to the avoidance of intrusiveness. We can achieve levels of privacy when the participants are using secure technological interventions that can mediate communication, and that can inspire the sense of being in control. People trust the interaction with the system when they feel in control of their privacy but without compromising the quality of the interaction.

Efficiency: Immediateness is crucial for the communication result of the system. As a result, we want the system to interact with the user quickly, on the spot.

Effectiveness: The majority of the participants lean towards a deep understanding of long-term behavioral patterns in contrast to data about the momentary emotional state of the participant. To support the designer's goals in empathizing with the user and in achieving a deeper understanding of the situation, we want the system to be able to map the participant's feelings and behavioral patterns over time.

Engagement: A more personal and informal interaction. A communication which is going to provide us with more in-depth insights. We want the system to support the designer in achieving these levels of personal involvement when interacting. 
Naturalness: An interaction that will feel natural to use. A technology that will be acceptable, thus enabling both parties to communicate instinctively. A naturalistic approach to the design of a system is one that supports a natural user experience.

Ease of Use: The most convenient and hassle-free interaction. We want the system to feel comfortable.

Information retrieval: To be assisted in avoiding information loss due to lack of memory as well as the system to store and retrieve data on the spot during the use of the system.

Self-Image awareness: The system supports different levels of self-presentation, supporting the designer in dealing with the situations of face-threatening acts in the communication.

Politeness: The system supports the designer to interact only when it matches the situation, "at the right time" and in the right manner.

Flexibility: The system dynamically adapts its needs based on the situation of use, supporting the user with the right tools and interactivity.

\subsubsection{Emotion Recognition Technologies}

Previous studies on real-life driving by Dobbins and Fairclough [66] have reported that the area of lifelogging has emerged as an application that is designed to continuously measure personal data with the purpose of supporting recall and self-reflection. Emotions can be captured continuously and in an unobtrusive manner. In our study, the participants do not trust the effectiveness of using face tracking, emotion recognition technologies, and in general psychophysiological techniques [67], due to technical and ethical considerations including false positives and effectiveness, privacy and safety. Even though the majority of our participants agree with the notion that it is the long-term user experience that matters [13], they are not primarily interested in momentary emotions especially coming from physiological measures. Thus, participants perceive mediums including video and audio for long-term behavioral observation, empathy building, and possible communication, as more effective and less intrusive forms of interaction.

\subsubsection{Unobtrusive Long-Term Behavioral Research}

As we previously mentioned, researchers in the automotive domain [66] have established the need for unobtrusive research. Furthermore, in-situ methods such as the Experience Sampling Method and the Day Reconstruction Method are increasingly applied in longitudinal settings, as Karapanos et al. [68] highlighted, while retrospective techniques offer a cost-effective alternative to longitudinal studies. Our results are in agreement with the theoretical framework of unobtrusive behavioral research in the automotive domain. The results indicate that the participants need to achieve a deeper understanding of the situation and map insights on feelings and long-term behavioral patterns, in other words, behavioral research which avoids intrusiveness by limiting their interaction with the driver/passenger.

The majority of our participants prefer a deeper understanding of the situation choosing holism versus reductionism and contextual data as previously defined by Roto et al. [69] to design for the new automotive experiences. Identifying the behavior of the users in the vehicle and their experience over time [70] was more critical to the designer's aims than identifying momentary emotions. An interesting finding is that UX designers are willing to design for experiences based on emotions as defined by [71] but not to draw inspiration by detected emotions since they perceive that they do not reflect on the overall experience.

\subsubsection{Empathy and Trust}

Our participants agree that trust in communication is essential and that it is achieved when the communication is natural, informal, transparent and high in social presence. They also expressed that the lack of a specific set of skills will result in impolite, unnatural and ineffective communication, hence a non-trustworthy interaction. As previous researchers highlight [60], trust as an experiential 
issue has been included in earlier approaches, but rarely as the primary objective of the design process. Trust has been an essential factor in many e-commerce user studies. Another important finding was that self-presentation issues need to be addressed since our participants are concerned about their appearance and how that will affect a possible communication with the driver/passenger or distract from the design task itself. In previous research [29], employees when teleconferencing were more aware of others' status and reactions, thereby were more cautious of their self-image and behaviors. Our participants believe that their self-presentation can affect the communication behavior and thus the driving experience itself. Even though we are designing for them, the participants empathize with the driver/passenger, and they only support/propose interventions that apply to all stakeholders of the system. Hence, the most compelling finding is that the participants believe that it is also crucial for the driver/passenger to find himself in control, valued, and interacting through the appropriate communication medium.

\subsection{UX Goals}

A user experience goal (UX goal) is an actionable insight for the development of products, services or systems. It describes the intended momentary emotion or the emotional relationship/bond that a person has with the designed product/service/system, as Lu and Roto [72] described. The same research also highlights that, in a multidisciplinary product development and marketing process, these concrete UX goals can be quite valuable since various stakeholder groups need to agree on what to design. Väätäjä et al. [73] considered a UX goal to be good when it guides design towards a positive experience, helps in communicating objectives, and is measurable. As a result of our study, we came up with actionable insights to communicate how to achieve a positive experience when designing for remote UX design systems in the automotive domain. These are UX goals, as presented below, that can inform the design of goal-directed personas and scenarios and the development of high fidelity prototypes. Designers use scenarios and personas to realize their users and their users' goals and place them in context. "A scenario is a concise description of a user using a software-based product to achieve a goal" [74] where the goals stem from the persona description. The prototype system or systems are then built based on the user scenarios of use.

The UX goals by themselves are general so as not to direct pre-defined design solutions. They only become more specific when connected with the needs that they fulfil and the context in which they need to be fulfilled: in our case, the automotive domain. Thus, we include the needs that must be fulfilled for each UX Goal in brackets, and we explain them in context. A list of the recommended UX goals when designing for relevant remote UX design systems in the Automotive domain is presented below:

1. Support the contextual understanding (Empathy and Effectiveness).

Supporting the contextual understanding in the car is a need that was also previously highlighted by Meschtscherjakov [5]. For example, given the mobility of the car, the context of it is dynamic and can only be compared with that of mobile devices. As a result, the affordances for understanding the context of a static home or office environment are not the same, and choosing the right ones will prove the effectiveness of the system. Since the form of transportation in this study is private, the car context is different concerning the social norms and relations among the passengers than when in a public environment. Building Empathy and Rapport as a need here differs from being able to afford it when in a public environment such as a train or even a library.

2. Reduce the intrusion of people's lives (Privacy).

Reducing the intrusion of people's lives in the automotive domain is a UX goal that can be afforded with various interventions. However, given the fact that the car will need to be connected in order for the remote system to work synchronously, some of the interventions that were previously applied in asynchronous settings are not applicable here.

3. Support long-term understanding of behavioral patterns (Effectiveness). 
In mobility and more specifically in transportation, where the metaphor of the customer journey comes from, users' behavior is identified by a sequence of events and not by an isolated moment as practitioners agreed. Support in identifying these patterns is a goal that will prove the system effective to practitioners when achieved.

4. Operate even when the problem is ill-defined (Flexibility and Effectiveness).

In many cases, designers have no set brief for designing a new product/service or system. Setting the brief, in this case in the automotive domain, requires flexibility to avoid the limitations that come with remote work. By being able to explore a situation without frustration or annoyance, the UX designer can effectively propose a design result. To support the designers to achieve these levels of effectiveness and flexibility, the system requires relevant affordances. We have previously highlighted that when designing for remote systems, the social context of the communication and the communication medium are of great importance. What is most important though is that the context has been found to influence the medium's perception and effectiveness. For example, even though telepresence is one of the most effective means of remote communication within the work environment, it might be neither flexible nor effective in a constantly changing mobile and private environment.

5. Avoid redundant information at any interaction (Efficiency, Ease of Use, and Flexibility).

The automotive context includes many spaces such as driver, front seat passenger, and rear seat passenger. As previously defined by Meschtscherjakov et al. [5] to capture the context holistically and gather insights, UX practitioners need to interact with a considerable amount of different data. The interplay between these data coming from these three different spaces should be usable and should fulfill the above-mentioned needs.

6. Avoid anxiety about uncertainty (Privacy, Engagement, Naturalness, and Self-image awareness).

Given the aforementioned limitations, including the dynamic and private nature of the automotive context, a supportive system should avoid making the non-automotive expert practitioner anxious about the quality of his work when interacting with it. Fulfilling the above-mentioned needs is crucial for the practitioner to interact smoothly and gather fruitful insights.

7. Feeling of intelligent interactivity (Efficiency, Ease of Use, and Information retrieval).

Intelligent interfaces are applied in many domains. Affordances for this UX goal should be common in relevant systems. However, the availability of these affordances does not mean that there is no need to fulfill these needs in the automotive context as well.

We learn that these UX Goals, excluding the increased privacy concern and the support of long-term behavioral patterns, are not exceptionally different from what we see in other domains where remote systems are applied. This welcomes opportunities to apply state-of-the-art practices and technologies from other domains for the implementation of the remote automotive UX system. We further recommend the design of compatible systems using the suggested UX Goals and their evaluation by automotive User experience researchers and designers which will shed light on automotive organization challenges for the adoption of such Research and Design Systems.

\section{Remote Design Trust and Privacy Relevance in Other Domains}

Characteristics such as clarity, naturalness, and communication etiquette to build trust and rapport in remote communication were previously investigated in telepresence systems' applications in organizations. Previous studies have also reported that a decrease in the degree of naturalness of a communication medium leads to increased cognitive effort; increased communication ambiguity; and decreased physiological arousal [62]. However, to build trust and rapport, one should consider that different types of technology require different forms of etiquette as previous studies have identified [75], and there are different kinds of etiquette for different settings [76]. Additionally, rules of etiquette depend on the social environment in which people use them. Similar to other norms, they are learned through experience in a community. Preece [76] emphasized this social dimension with the example of 
children who observe how adults and other children behave, absorb these norms, and, as a result, learn their community etiquette at an early age. Consequently, the cultural characteristics of a community influence the perceived politeness and naturalness of the remote interaction. In essence, politeness means "phrasing things in such a way to take into consideration the feelings of the others" [27].

Previous work on trust in automated vehicles [77] has identified the need to calibrate and understand trust. Scholars have long debated trust issues in other domains, for example, Metsctecherjakov et al. [78]; the findings of their studies could apply in the automotive domain. Studies in remote systems in organizations [79] suggest that the stage of the communication defines how trust is perceived and communicated: from the project starting-point to one week before the project mid-point, we identify that communication behaviors associated with trust are characterized by a combination of socially and task-oriented communications and the conveyance of enthusiasm. From the project mid-point to the project end, a sense of predictability in the interaction is generated by communication behaviors associated with trust.

Other researchers could further investigate the increased concern about privacy and safety in the automotive domain compared to other domains. Social situations in a car, compared to a personal situation, are a contextual metric that can alter the driving experience. For example, when designers and a user need to collaborate on a daily basis, one of the two parties intrudes the personal space of the other by having face-to-face (FTF) communication or computer-mediated communication (CMC) while being physically present in the first case or present but not physically present in the second case. However, anonymity is at the center of attention due to the general concern about privacy while using technology. It is more relevant in the absence of nonverbal cues which may lead to changes in the quality of the interaction including increased self-disclosure and intimacy in the same way that it provides more control over self-presentation [80]. Although the perceived social context of the in-vehicle situations may be the cause of increased privacy concern, this was not thoroughly investigated as part of our research, and we would recommend further exploration in this direction.

\section{Conclusions}

In this study, we co-designed a remote participatory automotive UX system. We increased active participation as a means to match the UX designer's needs with the affordances of remote UX design systems. To achieve that, we conducted individual co-design workshops with UX designers. These workshops also allowed us to identify their explicit, observable, tacit and latent needs based on empirical qualitative data. Needs including privacy, efficiency, effectiveness, engagement, naturalness, ease of use, information, retrieval, self-image awareness, politeness, and flexibility were identified and explained concerning the prototype system. We triangulated our data with the artifacts provided by the participants to translate these needs into UX goals. UX Goals that reflect UX designers' needs included trust and empathy building, privacy and self-image awareness, and holistic and behavioral long-term understandings of the user. We offer suggestions on future work to explore goal-directed personas and scenarios of use to inform practitioners on how to develop relevant future automotive UX systems.

Author Contributions: S.T. designed the process of the workshop, recruited the participants, designed the prototypes and facilitated the participatory design workshops. S.T. conducted the literature review for this study and both the thematic analysis and the glass wall analysis. M.P. helped review and edit the paper to its current form (as the supervisor of S.T.).

Funding: The research leading to these results has received funding from Brunel University London. S.T. received a bursary by Brunel University London as part of the Jaguar Land Rover funded Automotive Habitat Lab project (Research Code R33240).

Acknowledgments: The authors wish to thank Nick, Nicolas, Anna, and Maria for their help with recruiting. We are also very grateful to David for his comments on earlier drafts of the paper.

Conflicts of Interest: The authors declare no conflict of interest. The founding sponsors had no role in the design of the study; in the collection, analyses, or interpretation of data; in the writing of the manuscript, and in the decision to publish the results. 


\section{References}

1. Pfleging, B.; Rang, M.; Broy, N. Investigating user needs for non-driving-related activities during automated driving. In Proceedings of the 15th International Conference on Mobile and Ubiquitous Multimedia, Rovaniemi, Finland, 12-15 December 2016; ACM Press: New York, NY, USA, 2016; pp. 91-99.

2. Pettersson, I.; Ju, W. Design Techniques for Exploring Automotive Interaction in the Drive towards Automation. In Proceedings of the 2017 Conference on Designing Interactive Systems, Edinburgh, UK, 10-14 June 2017; pp. 147-160.

3. Meschtscherjakov, A.; Ju, W.; Tscheligi, M.; Szostak, D.; Krome, S.; Pfleging, B.; Ratan, R.; Politis, I.; Baltodano, S.; Miller, D.; et al. HCI and Autonomous Vehicles: Contextual Experience Informs Design. In Proceedings of the 2016 CHI Conference Extended Abstracts on Human Factors in Computing Systems, San Jose, CA, USA, 7-12 May 2016.

4. Tasoudis, S.; Perry, M. Mediated participatory design, for contextually aware in-vehicle experiences. In Proceedings of the 8th International Conference on Automotive User Interfaces and Interactive Vehicular Applications, Ann Arbor, MI, USA, 24-26 October 2016.

5. Meschtscherjakov, A.; Wilfinger, D.; Gridling, N.; Neureiter, K.; Tscheligi, M. Capture the car!: Qualitative in-situ methods to grasp the automotive context. In Proceedings of the 3rd International Conference on Automotive User Interfaces and Interactive Vehicular Applications, Salzburg, Austria, 30 November-2 December 2011; ACM Press: New York, NY, USA, 2011; p. 105.

6. Alvarez, I.; Rumbel, L.; Adams, R. Skyline: A rapid prototyping driving simulator for user experience. In Proceedings of the 7th International Conference on Automotive User Interfaces and Interactive Vehicular Applications, Nottingham, UK, 1-3 September 2015; ACM Press: New York, NY, USA, 2015; pp. 101-108.

7. Meschtscherjakov, A.; Wilfinger, D.; Osswald, S.; Perterer, N.; Tscheligi, M. Trip experience sampling: Assessing driver experience in the field. In Proceedings of the 4th International Conference on Automotive User Interfaces and Interactive Vehicular Applications, Portsmouth, NH, USA, 17-19 October 2012; ACM Press: New York, NY, USA, 2012; p. 225.

8. Niforatos, E.; Karapanos, E. EmoSnaps: A mobile application for emotion recall from facial expressions. Pers. Ubiquitous Comput. 2015, 19, 425-444. [CrossRef]

9. Martelaro, N.; Ju, W. WoZ Way: Enabling real-time remote interaction prototyping \& observation in on-road vehicles. In Proceedings of the 2017 ACM Conference on Computer Supported Cooperative Work and Social Computing, Portland, OR, USA, 25 February-1 March 2017; ACM Press: New York, NY, USA, 2017; pp. 21-24.

10. MacIntyre, B.; Gandy, M.; Dow, S.; Bolter, J.D. DART: A toolkit for rapid design exploration of augmented reality experiences. In Proceedings of the 17th Annual ACM Symposium on User Interface Software and Technology, Santa Fe, NM, USA, 24-27 October 2004; ACM Press: New York, NY, USA, 2004; p. 197.

11. Carter, S.; Mankoff, J.; Heer, J. Momento: Support for situated ubicomp experimentation. In Proceedings of the SIGCHI Conference on Human Factors in Computing Systems, San Jose, CA, USA, 30 April-3 May 2007; ACM Press: New York, NY, USA, 2007; p. 125.

12. Hassenzahl, M. Experience Design: Technology for All the Right Reasons. Synth. Lect. Hum.-Cent. Inf. 2010, 3, 1-95. [CrossRef]

13. Roto, V. User Experience from Product Creation Perspective. Towards a UX manifesto. In Proceedings of the 21st British HCI Group Annual Conference on People and Computers: HCI... But not as We Know It-Volume 2; Lancaster, UK, 2017; p. 37. Available online: https://pdfs.semanticscholar.org/3ae3/ 7d74a0aac4db3a5e232e7c83847f2e3a11ca.pdf (accessed on 31 July 2018).

14. Mekler, E.D.; Hornbæk, K. Momentary Pleasure or Lasting Meaning?: Distinguishing eudaimonic and hedonic user experiences. In Proceedings of the 2016 CHI Conference on Human Factors in Computing Systems, San Jose, CA, USA, 7-12 May 2016; ACM Press: New York, NY, USA, 2016; pp. 4509-4520.

15. Rittel, H.W.J.; Webber, M.M. Dilemmas in a general theory of planning. Policy Sci. 1973, 4, 155-169. [CrossRef]

16. Kenny, J. IDEO Design Thinking Toolkit For Educators. Available online: https:// designthinkingforeducators.com/design-thinking/ (accessed on 16 July 2017).

17. Addis, M.; Holbrook, M.B. On the conceptual link between mass customisation and experiential consumption: An explosion of subjectivity. J. Consum. Behav. 2001, 1, 50-66. [CrossRef] 
18. Hassenzahl, M.; Tractinsky, N. User experience-A research agenda. Behav. Inf. Technol. 2006, 25, 91-97. [CrossRef]

19. Sanders, E.B.N.; Stappers, P.J. Co-creation and the new landscapes of design. CoDesign 2008, 4, 5-18. [CrossRef]

20. Whiteside, J.; Wixon, D. Improving human-computer interaction-A quest for cognitive science. In Interfacing Thought: Cognitive Aspects of Human-Computer Interaction; MIT Press: Cambridge, MA, USA, 1987; pp. 353-365.

21. Schmidt, A.; Aidoo, K.; Takaluoma, A. Advanced interaction in context. In International Symposium on Handheld and Ubiquitous Computing; Springer: Berlin/Heidelberg, Germany, 1999.

22. Csikszentmihalyi, M. Flow and the Foundations of Positive Psychology; Springer: Dordrecht, The Netherlands, 2014.

23. Standaert, W.; Muylle, S.; Basu, A. An empirical study of the effectiveness of telepresence as a business meeting mode. Int. J. Inf. Technol. Manag. 2015, 17, 323-339. [CrossRef]

24. Derks, D.; Fischer, A.H.; Bos, A.E. The role of emotion in computer-mediated communication: A review. Comput. Hum. Behav. 2008, 24, 766-785. [CrossRef]

25. Cahir, J.; Lloyd, J. 'People just don't care': Practices of text messaging in the presence of others. Media Cult. Soc. 2015, 37, 703-719. [CrossRef]

26. Rhoads, M. Face-to-Face and Computer-Mediated Communication: What Does Theory Tell Us and What Have We Learned so Far? J. Plan. Lit. 2010, 25, 111-122. [CrossRef]

27. Morand, D.; Ocker, R. Politeness theory and computer-mediated communication: A sociolinguistic approach to analyzing relational messages. In Proceedings of the 36th Annual Hawaii International Conference on System Sciences, Big Island, HI, USA, 6-9 January 2003; p. 10.

28. Miller, C.A. Human-Computer Etiquette: Managing Expectations with Intentional Agents. Commun. ACM 2004, 47, 30. [CrossRef]

29. Park, N.; Rhoads, M.; Hou, J.; Lee, K.M.; Lai, J.Y.; Ulhas, K.R. Understanding the acceptance of teleconferencing systems among employees: An extension of the technology acceptance model. Comput. Hum. Behav. 2014, 39, 118-127. [CrossRef]

30. Visser, F.S.; Stappers, P.J.; van der Lugt, R.; Sanders, E.B.N. Contextmapping: Experiences from practice. CoDesign 2005, 1, 119-149. [CrossRef]

31. Cycil, C.; Perry, M.; Laurier, E.; Taylor, A. "Eyes free" in-car assistance: Parent and child passenger collaboration during phone calls. In Proceedings of the 15th International Conference on Human-Computer Interaction with Mobile Devices and Services, Munich, Germany, 27-30 August 2013; ACM Press: New York, NY, USA, 2013; p. 332.

32. Jordan, B.; Wasson, C. Autonomous Vehicle Study Builds Bridges between Industry and Academia. Ethnogr. Prax. Ind. Conf. Proc. 2015, 2015, 24-35. [CrossRef]

33. Gellatly, A.W.; Hansen, C.; Highstrom, M.; Weiss, J.P. Journey: General Motors' Move to Incorporate Contextual Design Into Its Next Generation of Automotive HMI Designs. In Proceedings of the 2nd International Conference on Automotive User Interfaces and Interactive Vehicular Applications, Pittsburgh, PA, USA, 11-12 November 2010; ACM Press: New York, NY, USA, 2010; p. 156.

34. Jordan, B.; Wasson, C.; Roth-Lobo, H. Ethnographic Study Lifts the Hood on what REALLY Goes on Inside That Car, Ethnographic Study Lifts the Hood on What REALLY Goes on Inside That Car. EPIC/Advancing the Value of Ethnography in Industry. Available online: https:/ / www.epicpeople.org/ethnographic-studylifts-the-hood/\# (accessed on 14 March 2016).

35. Bartl, M.; Jawecki, G.; Wiegandt, P. Co-Creation in New Product Development: Conceptual Framework and Application in the Automotive Industry. In Proceedings of the R\&D Management Conference-Information, Imagination and Intelligence, Manchester, UK, 30 June-2 July 2010.

36. Ross, J. The Role of Observation in User Research: UXmatters. Available online: https:/ / www.uxmatters. $\mathrm{com} / \mathrm{mt} /$ archives / 2018/09/the-role-of-observation-in-user-research.php (accessed on 20 October 2018).

37. Six, J.M. Tools for Remote, Collaborative UX Design: UXmatters. Available online: https:/ /www.uxmatters. com/mt/archives/2017/11/tools-for-remote-collaborative-ux-design.php (accessed on 20 October 2018).

38. Wilfinger, D.; Murer, M.; Baumgartner, A.; Döttlinger, C.; Meschtscherjakov, A.; Tscheligi, M. The car data toolkit: Smartphone supported automotive HCI research. In Proceedings of the 5th International Conference on Automotive User Interfaces and Interactive Vehicular Applications, Eindhoven, The Netherlands, 28-30 October 2013; ACM Press: New York, NY, USA, 2013; pp. 168-175. 
39. Rizzo, F. Co-design versus User Centred Design: Framing the differences. In Notes on Doctoral Research in Design; Franco Angeli: Milano, Italy, 2011; p. 125.

40. Frauenberger, C.; Good, J.; Keay-Bright, W.; Pain, H. Interpreting input from children: A designerly approach. In Proceedings of the 2012 ACM Annual Conference on Human Factors in Computing Systems, Austin, TX, USA, 5-10 May 2012; ACM Press: New York, NY, USA, 2012; p. 2377.

41. Lindsay, S.; Brittain, K.; Jackson, D.; Ladha, C.; Ladha, K.; Olivier, P. Empathy, participatory design and people with dementia. In Proceedings of the 2012 ACM annual conference on Human Factors in Computing Systems, Austin, TX, USA, 5-10 May 2012; ACM Press: New York, NY, USA, 2012; p. 521.

42. Halskov, K.; Dalsgård, P. Inspiration card workshops. In Proceedings of the 6th ACM Conference on Designing Interactive Systems, University Park, PA, USA, 26-28 June 2006; ACM Press: New York, NY, USA, 2006; p. 2.

43. Uzor, S.; Baillie, L.; Skelton, D. Senior designers: Empowering seniors to design enjoyable falls rehabilitation tools. In Proceedings of the 2012 ACM annual conference on Human Factors in Computing Systems, Austin, TX, USA, 5-10 May 2012; ACM Press: New York, NY, USA, 2012; p. 1179.

44. Vines, J.; Blythe, M.; Lindsay, S.; Dunphy, P.; Monk, A.; Olivier, P. Questionable concepts: Critique as resource for designing with eighty somethings. In Proceedings of the 2012 ACM Annual Conference on Human Factors in Computing Systems, Austin, TX, USA, 5-10 May 2012; ACM Press: New York, NY, USA, 2012; p. 1169.

45. Hook, J.; Green, D.; McCarthy, J.; Taylor, S.; Wright, P.; Olivier, P. A VJ centered exploration of expressive interaction. In Proceedings of the 2011 Annual Conference on Human Factors in Computing Systems, Vancouver, BC, Canada, 7-12 May 2011; ACM Press: New York, NY, USA, 2011; p. 1265.

46. Le Dantec, C. Participation and publics: Supporting community engagement. In Proceedings of the 2012 ACM Annual Conference on Human Factors in Computing Systems, Austin, TX, USA, 5-10 May 2012; ACM Press: New York, NY, USA, 2012; p. 1351.

47. Vines, J.; Blythe, M.; Dunphy, P.; Vlachokyriakos, V.; Teece, I.; Monk, A.; Olivier, P. Cheque mates: Participatory design of digital payments with eighty somethings. In Proceedings of the 2012 ACM annual Conference on Human Factors in Computing Systems, Austin, TX, USA, 5-10 May 2012; ACM Press: New York, NY, USA, 2012; p. 1189.

48. Balaam, M.; Ricketts, I.; Mawson, S.; Burridge, J.; Rennick Egglestone, S.; Fitzpatrick, G.; Rodden, T.; Hughes, A.M.; Wilkinson, A.; Nind, T.; et al. Motivating mobility: Designing for lived motivation in stroke rehabilitation. In Proceedings of the 2011 Annual Conference on Human Factors in Computing Systems, Vancouver, BC, Canada, 7-12 May 2011; ACM Press: New York, NY, USA, 2011; p. 3073.

49. Vines, J.; Clarke, R.; Wright, P.; McCarthy, J.; Olivier, P. Configuring participation: On how we involve people in design. In Proceedings of the SIGCHI Conference on Human Factors in Computing Systems, Paris, France, 27 April-2 May 2013; p. 429.

50. Muller, M.J. Participatory design: The third space in HCI. In Human-Computer Interaction: Development Process; Erlbaum Associates Inc.: Mahwah, NJ, USA, 2003; pp. 1051-1068.

51. Sanders, E.B.N.; Stappers, P.J. Probes, toolkits and prototypes: Three approaches to making in codesigning. CoDesign 2014, 10, 5-14. [CrossRef]

52. Tonetto, L.M.; Desmet, P.M. Why we love or hate our cars: A qualitative approach to the development of a quantitative user experience survey. Appl. Ergon. 2016, 56, 68-74. [CrossRef] [PubMed]

53. McCarthy, J.; Wright, P. Technology as experience. Interactions 2004, 11, 42. [CrossRef]

54. Sanders, E.B.N.; Brandt, E.; Binder, T. A framework for organizing the tools and techniques of participatory design. In Proceedings of the 11th Biennial Participatory Design Conference, Sydney, Australia, 29 November-3 December 2010; ACM Press: New York, NY, USA, 2010; p. 195.

55. Sleeswijk Visser, F.; van der Lugt, R.; Stappers, P.J. Sharing User Experiences in the Product Innovation Process: Participatory Design Needs Participatory Communication. Creat. Innov. Manag. 2007, 16, 35-45. [CrossRef]

56. Sanders, E.B.N. From User-Centered to Participatory Design Approaches. In Design and the Social Sciences; CRC Press: Boca Raton, FL, USA, 2003; pp. 18-25.

57. Iversen, O.S.; Halskov, K.; Leong, T.W. Rekindling values in participatory design. In Proceedings of the 11th Biennial Participatory Design Conference, Sydney, Australia, 29 November-3 December 2010; ACM Press: New York, NY, USA, 2010; p. 91. 
58. Baskerville, R.; Pries-Heje, J.; Venable, J. Soft design science methodology. In Proceedings of the 4th International Conference on Design Science Research in Information Systems and Technology, Philadelphia, PA, USA, 7-8 May 2009; ACM Press: New York, NY, USA, 2009; p. 1.

59. Lucero, A. Using Affinity Diagrams to Evaluate Interactive Prototypes. In Human-Computer Interaction; Springer: Cham, Switzerland, 2015; pp. 231-248.

60. Kaasinen, E.; Roto, V.; Hakulinen, J.; Heimonen, T.; Jokinen, J.P.P.; Karvonen, H.; Keskinen, T.; Koskinen, H.; Lu, Y.; Saariluoma, P.; et al. Defining user experience goals to guide the design of industrial systems. Behav. Inf. Technol. 2015, 34, 976-991. [CrossRef]

61. Bevan, N. Classifying and selecting UX and usability measures. In Proceedings of the International Workshop on Meaningful Measures: Valid Useful User Experience Measurement, Reykjavik, Iceland, 18 June 2008; pp. 13-18.

62. Kock, N. Media Richness or Media Naturalness? The Evolution of Our Biological Communication Apparatus and Its Influence on Our Behavior toward E-Communication Tools. IEEE Trans. Prof. Commun. 2005, 48, 117-130. [CrossRef]

63. Eckoldt, K.; Hassenzahl, M.; Laschke, M.; Knobel, M. Alternatives: Exploring the car's design space from an experience-oriented perspective. In Proceedings of the 6th International Conference on Designing Pleasurable Products and Interfaces, Newcastle upon Tyne, UK, 3 -5 September 2013; ACM Press: New York, NY, USA, 2013; p. 156.

64. Körber, M.; Bengler, K. Measurement of momentary user experience in an automotive context. In Proceedings of the 5th International Conference on Automotive User Interfaces and Interactive Vehicular Applications, Eindhoven, The Netherlands, 28-30 October 2013; ACM Press: New York, NY, USA, 2013; pp. 194-201.

65. Kujala, S.; Roto, V.; Väänänen-Vainio-Mattila, K.; Karapanos, E.; Sinnelä, A. UX Curve: A method for evaluating long-term user experience. Interact. Comput. 2011, 23, 473-483. [CrossRef]

66. Dobbins, C.; Fairclough, S. A mobile lifelogging platform to measure anxiety and anger during real-life driving. In Proceedings of the 2017 IEEE International Conference on Pervasive Computing and Communications Workshops (PerCom Workshops), Kona, HI, USA, 13-17 March 2017; pp. 327-332.

67. Mandryk, R.L.; Inkpen, K.M.; Calvert, T.W. Using psychophysiological techniques to measure user experience with entertainment technologies. Behav. Inf. Technol. 2006, 25, 141-158. [CrossRef]

68. Karapanos, E.; Jain, J.; Hassenzahl, M. Theories, methods and case studies of longitudinal HCI research. In Proceedings of the 2012 ACM Annual Conference Extended Abstracts on Human Factors in Computing Systems Extended Abstracts, Austin, TX, USA, 5-10 May 2012; ACM Press: New York, NY, USA, 2012; p. 2727.

69. Roto, V.; Väätäjä, H.; Jumisko-Pyykkö, S.; Väänänen-Vainio-Mattila, K. Best practices for capturing context in user experience studies in the wild. In Proceedings of the 15th International Academic MindTrek Conference on Envisioning Future Media Environments, Tampere, Finland, 28-30 September 2011; ACM Press: New York, NY, USA, 2011; p. 91.

70. Karapanos, E.; Zimmerman, J.; Forlizzi, J.; Martens, J.B. User experience over time. In Proceedings of the 27th International Conference on Human Factors in Computing Systems, Boston, MA, USA, 4-9 April 2009; ACM Press: New York, NY, USA, 2009; p. 729.

71. Desmet, P.; Hekkert, P. Framework of Product Experience Human-Product Interaction. Int. J. Des. Nat. 2007, 30, 57-66.

72. Lu, Y.; Roto, V. Towards meaning change: Experience Goals Driving Design Space Expansion. In Proceedings of the 8th Nordic Conference on Human-Computer Interaction Fun, Fast, Foundational, Helsinki, Finland, 26-30 October 2014; ACM Press: New York, NY, USA, 2014; pp. 717-726.

73. Väätäjä, H.; Olsson, T.; Savioja, P.; Roto, V. UX Goals 2012 workshop-How to utilize user experience goals in design? In Proceedings of the Workshop Held in Conjunction with NordiCHI 2012, Copenhagen, Denmark, 14 October 2012.

74. Hevner, A.R.; Chatterjee, S. Design Research in Information Systems: Theory and Practice; Springer Science and Business Media: New York, NY, USA, 2010; p. 127.

75. George, J.F.; Carlson, J.R.; Valacich, J.S. Media selection as a strategic component of communication. MIS Q. 2013, 37, 1233-1252. [CrossRef]

76. Preece, J. Etiquette online. Commun. ACM 2004, 47, 56. [CrossRef] 
77. Mirnig, A.G.; Trösterer, S.; Meschtscherjakov, A.; Gärtner, M.; Tscheligi, M. Trust in Automated Vehicles. i-com 2018, 17, 79-90. [CrossRef]

78. Meschtscherjakov, A.; Tscheligi, M.; Pfleging, B.; Sadeghian Borojeni, S.; Ju, W.; Palanque, P.; Riener, A.; Mutlu, B.; Kun, A.L. Interacting with Autonomous Vehicles: Learning from other Domains. In Proceedings of the Extended Abstracts of the 2018 CHI Conference on Human Factors in Computing Systems, Montreal, QC, Canada, 21-26 April 2018; ACM Press: New York, NY, USA, 2018; pp. 1-8.

79. Rico, R.; Alcover, C.M.; Sanchez-Manzanares, M.; Gil, F. The joint relationships of communication behaviors and task interdependence on trust building and change in virtual project teams. Soc. Sci. Inf. 2009, 48, 229-255. [CrossRef]

80. Shalom, J.G.; Israeli, H.; Markovitzky, O.; Lipsitz, J.D. Social anxiety and physiological arousal during computer mediated vs. face to face communication. Comput. Hum. Behav. 2015, 44, 202-208. [CrossRef]

(C) 2018 by the authors. Licensee MDPI, Basel, Switzerland. This article is an open access article distributed under the terms and conditions of the Creative Commons Attribution (CC BY) license (http://creativecommons.org/licenses/by/4.0/). 